\title{
Dislocation Velocity in Single and Polycrystalline Silicon-Iron*
}

\author{
D. W. Moon† and T. VReEland, JR. \\ W. M. Keck Laboratory of Engineering Materials, California Institute of Technology, Pasadena, California
}

(Received 19 July 1967)

\begin{abstract}
The stress dependence of screw-dislocation velocity in single and polycrystalline specimens of an iron$3.14 \%$ silicon alloy was measured by observation of slip-band growth. An electrolytic etching technique was used to reveal dislocation intersections with the specimen surface, and slip bands were observed to form from fresh scratches and from grain boundaries as a result of pulse loading. Screw dislocation velocity on the $\{110\}\langle 111\rangle$ system in single crystals at room temperature followed the relation $v=\left(\tau / \tau_{0}\right)^{n}$, where $n=30.1$. A plot of screw-dislocation velocity vs nominal resolved shear stress in individual grains of polycrystalline specimens shows considerable scatter which is attributed to the effects of stress variations due to elastic anisotropy. Observation of slip-band growth in scatched and unscratched grains indicates that the stress required to activate grain boundary sources is greater than the stress required to propagate fresh dislocations.
\end{abstract}

\section{INTRODUCTION}

The mobility of edge dislocations in silicon-iron was first studied by Stein and Low. ${ }^{1}$ They investigated the stress and temperature dependence of the growth of slip bands on the $\{110\}\langle 111\rangle$ system in single-crystal specimens. Erickson ${ }^{2}$ investigated dislocation mobility on the $\{112\}\langle 111\rangle$ system. Their results show that edge dislocation velocity is a very sensitive function of stress in silicon-iron which contains sufficient carbon for the Dunn and Daniels etching technique ${ }^{3}$ to be effective.

Both dislocation locking by interstitial impurities and dislocation dynamics have been used to explain various aspects of the yield phenomenon in bcc materials. The earliest dislocation theories of a distinct yield point were based upon the concept of Cottrell locking of dislocations by interstitial impurities. The yield point drop was attributed to the first break-away of dislocations. Johnston and Gilman ${ }^{5}$ and Johnston ${ }^{6}$ demonstrated that a yield point drop in single crystals can result from the kinetics of dislocation multiplication and motion. $\mathrm{Hahn}^{7}$ and Cottrell ${ }^{8}$ applied the dislocation kinetics theory to the yielding of polycrystalline materials. Neither of these treatments considered the heterogeneous nature of the polycrystalline material. The results of the study reported herein give information on which a more quantitative treatment of dislocation dynamics in polycrystalline materials may be based.

\footnotetext{
* This work was sponsored by the U.S. Atomic Energy Commission.

$\dagger$ Present address: Depratment of Mechanical Engineering, University of California, Davis, Calif.

1 D. F. Stein and J. R. Low, Jr., J. Appl. Phys. 31, 362 (1960).

2 J. S. Erickson, J. Appl. Phys. 33, 2499 (1962).

${ }^{3}$ C. G. Dunn and F. W. Daniels, Transactions [American Institute of Mining (Metall.) Engineers, 1951], p. 147.

${ }_{4}^{4}$ A. H. Cottrell, Symposium on the Plastic Deformation of Crystalline Solids (Mellon Institute, 1950), p. 60.

SW. G. Johnston and J. J. Gilman, J. Appl. Phys. 30, 129 (1959).

${ }_{6}^{6}$ W. G. Johnston, J. Appl. Phys. 33, 2716 (1962).

${ }^{7}$ G. T. Hahn, Acta Met. 30, 727 (1962).

8 A. H. Cottrell, The Relation between the Structure and Mechanical Properties of Metals (Her Majesty's Stationery Office, London, 1963), p. 456 .
}

Conflicting conclusions regarding the source of dislocations in polycrystalline silicon-iron appear in the literature. Suits and Chalmers ${ }^{9}$ concluded that the sources of mobile dislocations are local stress concentrations which are distributed throughout the volume of each grain. They identify the stress required to produce slip bands across grains with the stress required to produce a sufficiently high dislocation velocity. Worthington and Smith $^{10}$ observed that the grain boundaries were the primary source of mobile dislocations in extremely coarse-grained, fully annealed material. They also concluded that the stress necessary to generate dislocations is less than the stress necessary to move dislocations across grains. This paper presents evidence related to the question of the relative importance of dislocation sources within the grain and sources at or near the grain boundaries, and to the relative importance of source activation and dislocation mobility in the formation of slip bands.

\section{MATERIAL AND TEST PROCEDURES}

The General Electric Research Laboratory kindly supplied the material used in this investigation. Table I lists the composition of the alloy, which is from the same heat as that used by Suits and Chalmers. ${ }^{9}$

Suits and Low ${ }^{11}$ have reported that an iron-silicon alloy must contain a carbon content from 0.004 to 0.006 wt $\%$ in order for dislocation sites to be revealed by the Dunn and Daniels etching technique. ${ }^{3}$ Consequently, the as-received strip stock was gas carburized to increase the carbon content to approx $0.005 \mathrm{wt} \%$.

Polycrystalline specimens with gauge sections $\frac{1}{2} \mathrm{~mm}$ thick, $6.35 \mathrm{~mm}$ wide, and $25 \mathrm{~mm}$ long were prepared from the carburized material which had been cold rolled to a $6 \%$ reduction in area. Recrystallization for $1 \mathrm{~h}$ at $1000^{\circ} \mathrm{C}$ produced an average grain diameter of $76 \mu$ (an average of 6.7 grains through the $\frac{1}{2}$-mm thickness). A dislocation etch pit density of $10^{6}-10^{7} \mathrm{~cm}^{-2}$ was

${ }^{9}$ J. C. Suits and B. Chalmers, Acta Met. 9, 854 (1961),

${ }_{10}$ P. J. Worthington and E. Smith, Acta Met. 12, 1277 (1964).

11 J. C. Suits and J. R. Low, Jr., Acta Met. 5, 285 (1957). 
observed within the grains. Ordinary stress-strain curves for typical specimens are shown in Fig. 1.

Single-crystal specimens were produced by the strainanneal method of Dunn and Nonken. ${ }^{12}$ The as-grown crystals did not etch properly to reveal dislocations. There were no etch figures in the single crystal except for the region within $3 \mathrm{~mm}$ of the remnant polycrystalline portion of the specimen which had not passed through the temperature gradient into the hot zone of the furnace. Within this small portion of the single crystal, etching occurred, except in the boundaries of a cell pattern closely resembling the adjacent polycrystalline grain structure. Furthermore, cell walls in the single crystal were aligned with grain boundaries in the polycrystalline region.

This etching behavior may be explained as follows: The polycrystalline material contains a substantial amount of carbon in the grain boundaries which does not contribute to the dislocation etching behavior. When the polycrystal is converted into a single crystal, this grain boundary carbon is released so that the carbon content of the single crystal is greater than the maximum amount for proper etching of dislocations. In the region of the single crystal immediately adjacent to the polycrystalline material, the former grain boundary carbon has not had sufficient diffusion time to become uniformly distributed and hence the etching reveals a "ghost" image of the former grain boundaries.

This unsatisfactory etching behavior of the singlecrystal specimens was corrected by first decarburizing them and then recarburizing to provide the correct carbon content for dislocation etching. The decarburization was accomplished by heating the crystals for $24 \mathrm{~h}$ at $680^{\circ} \mathrm{C}$ in an atmosphere of hydrogen plus water vapor. Recarburization to $0.005 \mathrm{wt} \%$ carbon was accomplished by heating for $10 \mathrm{~h}$ at $835^{\circ} \mathrm{C}$ in an atmosphere of dry hydrogen plus $1 \%$ by volume of methane. Dislocations in the single crystals etched in the proper

TABle I. Composition of test material.

\begin{tabular}{ll}
\hline \multicolumn{1}{c}{ Element } & wt \% \\
\hline Silicon & 3.14 \\
Carbon & 0.001 \\
Manganese & 0.043 \\
Phosphorous & 0.008 \\
Sulphur & 0.018 \\
Nickel & 0.066 \\
Copper. & 0.079 \\
Tin & 0.010 \\
Chromium & trace \\
Aluminum & trace \\
Iron & remainder \\
\hline
\end{tabular}

${ }^{12}$ C. G. Dunn and G. C. Nonken, Metal Progr. 64, 71 (1953).
FIG. 1. Ordinary stress-strain curves for a typical polycrystal specimen and single-crystal specimen S1. Crosshead speed on Instron: $0.025 \mathrm{~cm} / \mathrm{min}$.

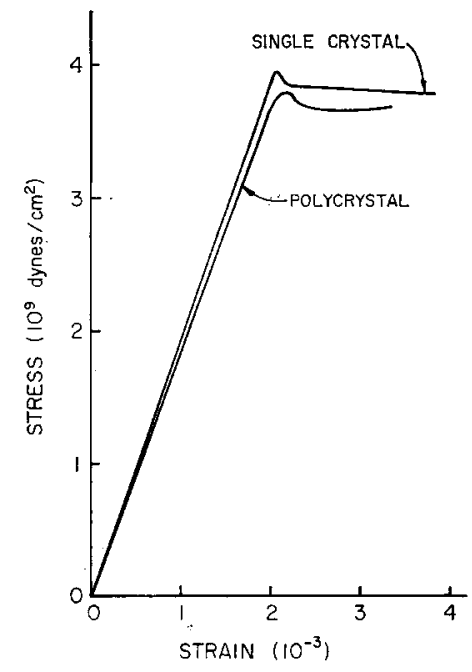

manner following these treatments, and the dislocation etch pit density was $10^{6}-10^{7} \mathrm{~cm}^{-2}$. The single crystals were glued to loading tabs to form test specimens with essentially the same dimensions as the polycrystalline specimens. The orientation of the single-crystal specimens is shown in Fig. 2.

A source of fresh dislocations was introduced to both single-crystal and polycrystalline specimens by scratching a polished surface of the gauge section with a conical diamond stylus, using a load of $3.6 \mathrm{~g}$. The specimens were pulsed loaded within 5 min of scratching.

Pulse loading of the thin tensile specimens was accomplished using special fixtures designed to minimize bending strains. The bending stress was limited to $1 \%$ of the tensile stress by these fixtures. A rapid loading machine was used to apply square load pulses with a load rise and fall time of 2 msec..$^{13} \mathrm{~A}$ record of load vs time was recorded on a galvanometer type oscillograph.

Electrolytic etching ${ }^{6}$ immediately after load application reveals only the dislocation sites which existed prior to loading. Specimens were aged for $1 \mathrm{~h}$ at $145^{\circ} \mathrm{C}$ and then electrolytically etched to reveal the dislocation configuration produced by the loading.

The orientation of selected individual grains in the polycrystalline specimens was determined by a modification of the method described by Barrett. ${ }^{14}$ The nominal resolved shear stress in those grains produced by the pulse load could then be calculated. Etch pits having plane faces parallel to $\{100\}$ planes were produced by etching with a $25 \%$ solution of nitric acid in water. The direction of the normal to one such $\{100\}$ etch pit face, i.e., a $\langle 100\rangle$ direction with respect to the tensile axis was determined by means of a Unitron microgoniometer microscope. A second $\langle 100\rangle$ direction could not be

${ }^{13}$ T. L. Russell, D. S. Wood, and D. S. Clark, Acta Met. 9, 1054 (1961).

${ }^{14}$ C. S. Barrett, Structure of Metals (McGraw-Hill Book Company, Inc., New York, 1952), p. 193. 


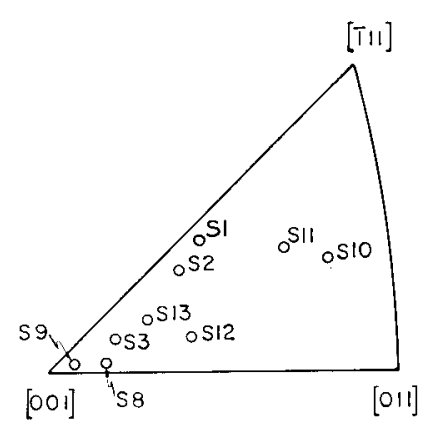

determined in this manner because the range of one of the Unitron goniometer circles is limited to $90^{\circ}$. The orientation of the crystal axes of the selected grain with respect to the tensile axis was completed by an observation of the orientation of the trace of a $\{100\}$ slip plane on the specimen surface together with a consideration of the resolved shear stress acting on all six of the $\{110\}\langle 111\rangle$ slip systems which were compatible with the observed slip trace. The grain orientation was assumed to be that orientation for which the observed slip trace would be produced by the $\{110\}$ $\langle 111\rangle$ slip system which was subjected to the maximum resolved shear stress.

The observed dislocation velocity was taken to be the length of the longest slip lines extending from the scratch, divided by the duration of the load pulse. Data was taken in polycrystalline specimens, from 48 grains in which the slip lines did not reach a grain boundary.

Multiple tests were performed on most of the singlecrystal specimens. Aging of the specimens after each load pulse anchored the fresh dislocations, and new scratches were used on subsequent tests, until no unslipped areas of the specimens remained.

\section{EXPERIMENTAL METHOD}

\section{Single Crystals}

The dislocation configuration after a pulse load on single-crystal specimen $\mathrm{S} 9$ is shown in Fig. 3. Slip traces observed on the flat surfaces and edges of the specimen gauge section were used to determine the Miller indices of the slip planes. The specimens on which traces could be observed on the flat surface and edge clearly slipped on $\{110\}$ planes. A further analysis was required when only one slip trace could be clearly identified. Assuming a $\langle 111\rangle$ slip direction, it follows that the maximum number of "possible" slip planes consistent with a single slip trace is four. The resolved shear stress for each of these "possible" slip systems was calculated, and in each case the system subjected to the highest resolved shear stress was of the $\{110\}\langle 111\rangle$ type. Attempts to obtain observed velocities higher than $2.8 \times 10^{-2} \mathrm{~cm} / \mathrm{sec}$. were unsuccessful due to twinning or cleavage of the specimens. The twin systems observed and their role in nucleating cracks is consistent with previous reports by Hull. ${ }^{15}$

The velocity of slip-band growth in the direction perpendicular to the Burgers vector (screw-dislocation velocity) is obtained by multiplying the observed velocity by the sine of the angle between the Burgers vector and the slip trace. This velocity is plotted vs the resolved shear stress in Fig. 4. The screw-dislocation velocity vs resolved shear-stress data for the single crystals follows the relation

$$
v=v_{0}\left(\tau / \tau_{0}\right)^{n},
$$

where $v_{0}=1 \mathrm{~cm} / \mathrm{sec}, \tau_{0}=2.30 \times 10^{9} \mathrm{dyn} / \mathrm{cm}^{2}$ and $n=30.1$.

\section{Polycrystalline Specimens}

The dislocation configuration in a scratched and aged polycrystalline specimen is shown in Fig. 5. A typical area of a scratched, loaded, and aged specimen is shown in Fig. 6. The majority of scratched grains show some slip-line activity emanating from the scratch, and a relatively small number of unscratched grains show slip bands which generally traverse the entire grain. Screwdislocation velocity was obtained from the observed velocity along the slip trace as explained above. Screwdislocation velocity vs the nominal resolved shear stress in the grain is plotted in Fig. 4. Considerable scatter in the data for the polycrystalline specimens may be seen in Fig. 4, and the data points all lie above those for the single crystals.

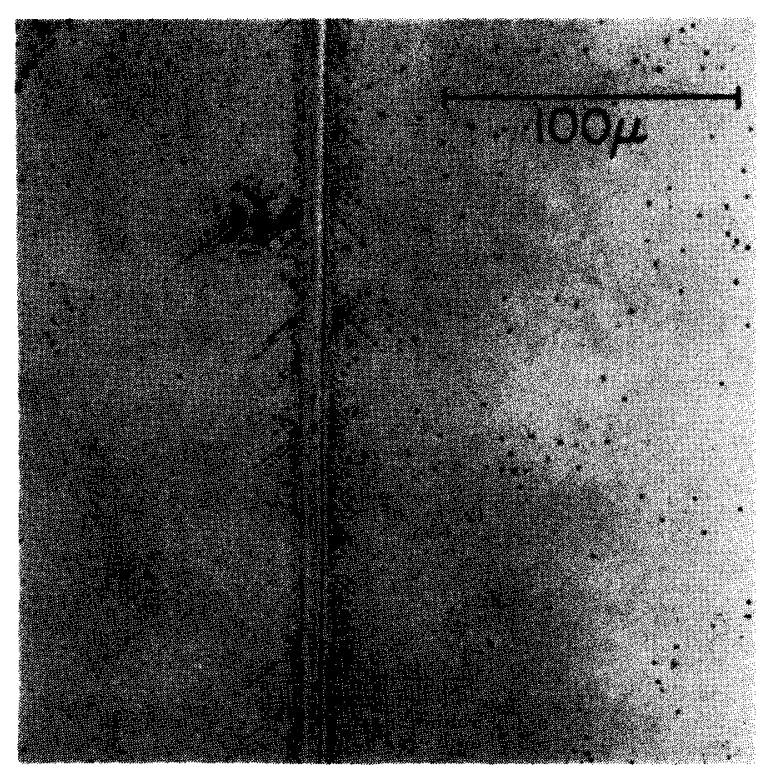

FIG. 3. Dislocation configuration after a pulse load in singlecrystal specimen S9.

${ }^{15}$ D. Hull, Proc. Roy. Soc. (London) 27A, 5 (1963). 


\section{DISCUSSION}

\section{Dislocation Velocity}

The velocity of edge dislocations in the slip bands observed by Stein and Low ${ }^{1}$ is shown by the dashed line in Fig. 4. The mobility exponent $n$ is essentially the same, but $\tau_{0}$ is different than that found in this investigation. The factors responsible for the difference are most probably the dislocation orientation and to a lesser extent the initial dislocation density.

Low and Guard ${ }^{16}$ have shown that dislocation loops in silicon-iron are very much elongated in the direction of the Burgers vector. This indicates that the velocity of screw-oriented dislocations is much less than the velocity of edge-oriented dislocations at the same resolved stress, as is seen in Fig. 4. A somewhat lower dislocation velocity might be expected in the specimens tested in this investigation, since they contained a higher initial dislocation density $\left(\approx 10^{6} \mathrm{~cm}^{-2}\right)$ than the specimens tested by Stein and Low $\left(\approx 10^{5} \mathrm{~cm}^{-2}\right)$.

A relatively large deviation from a linear relationship between $\log v$ and $\log \tau$ is found in the polycrystalline data. The initial density of dislocations and the chemical composition of both single-crystal and polycrystalline material were nearly equal. Therefore, the scatter of the polycrystalline data must be due to deviations in the resolved shear stress on the slip systems from that calculated from the tensile stress and the Schmid factor. The deviations are attributed to the effect of elastic anisotropy on the actual stress distribution in the poly-

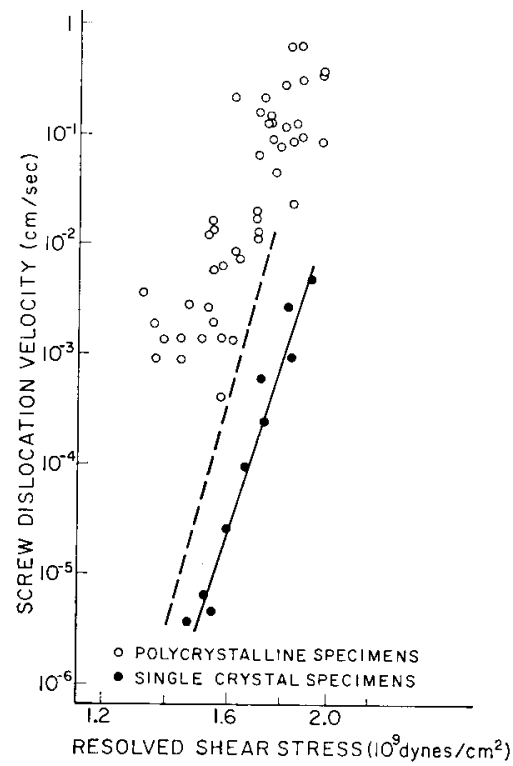

Fig. 4. Stress dependence of screw-dislocation velocity in single and polycrystalline specimens. Edge-dislocation velocity from the data of Stein and Low is shown as a dashed line.

${ }^{16} \mathrm{~J}$. R. Low and R. W. Guard, Acta Met. 7, 171 (1959).

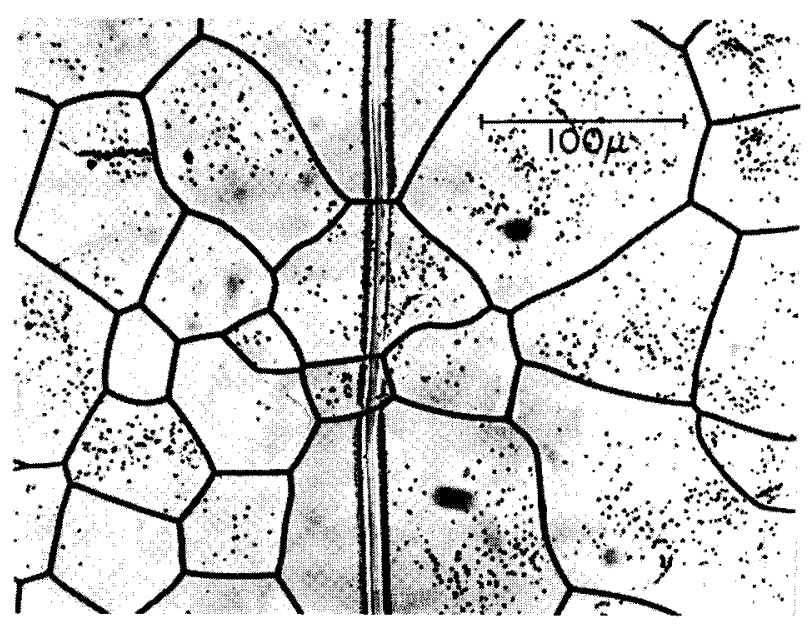

FIG. 5. Typical dislocation configuration in a scratched and aged specimen.

crystalline specimens. A maximum deviation of $30 \%$ in stress is observed between the results obtained with the single-crystal and polycrystalline specimens. This deviation is reasonable in view of the approx $40 \%$ variation in elastic properties of $\mathrm{Fe}$ with crystal orientation. ${ }^{17}$

All data points based upon the tests with polycryștalline specimens lie above the curve for data on singlecrystal specimens. This is because the duration of the stress pulse used to determine $v$-vs- $\tau$ in polycrystalline specimens was such that data were obtained only in the highly stressed grains. A relatively short-duration pulse test at a constant $\tau$ produces data from only those grains containing the highest resolved shear stress. Longer duration pulses will allow the slip bands to traverse the most highly stressed grains. The pulse duration was chosen so that very few grains were completely traversed. Data were therefore obtained from only the most highly stressed grains.

The dislocation mobility data in polycrystalline silicon-iron may be used in a quantitative treatment of yielding and the delay time for yielding under constant stress. This treatment will be presented in a subsequent paper.

\section{Dislocation Sources}

The majority of slip bands were observed to form from fresh scratches. A few slip bands formed from what appeared to be inclusions in the single crystals and from grain boundaries in the polycrystals. The shape of all slip bands which did not traverse the grain indicated that the bands initiated at grain boundaries. The distribution of individual etch figures within the bands shows a decreasing density of dislocations with increasing distance from the grain boundary. This is also

${ }^{17}$ D. McLean, Mechanical Properties of Metals (John Wiley \& Sons, Inc., New York, 1962), p. 6. 


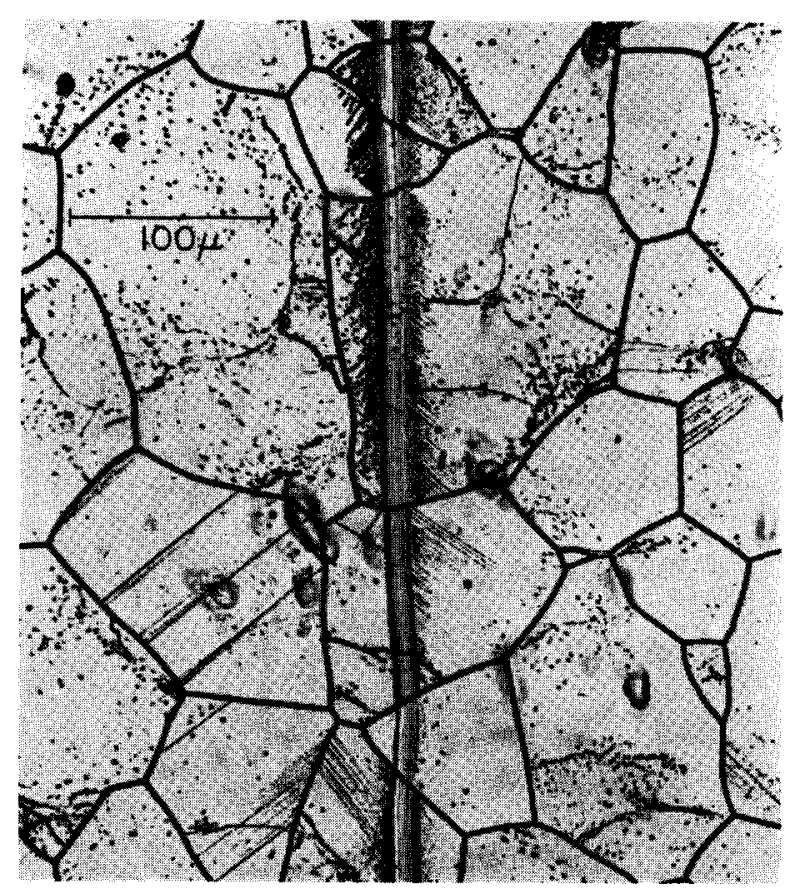

FrG. 6. Typical dislocation configuration in scratched, pulseloaded, and aged specimen T14.

seen in the slip bands emanating from fresh scratches. Slip-band growth by the cross-slip mechanism proposed by Koehler ${ }^{18}$ should produce bands with dislocation densities which decrease as the distance from the source increases. If the primary sources of dislocations were uniformly distributed throughout the matrix as suggested by Suits and Chalmers, ${ }^{9}$ then at least some evidence should be obtained in which both ends of a slip band terminate within a grain. This would especially be true for specimens subjected to short-duration pulse loading. No such slip bands were observed in this investigation, so the grain boundaries were the primary sources as reported by Worthington and Smith.10

Suits and Chalmers and Worthington and Smith suggested that dislocation mobility limited the formation of the slip bands they observed in constant strainrate tests. Suits and Chalmers used the velocity data of Stein and Low to show that dislocations would cross

\footnotetext{
${ }^{18}$ J. S. Koelher, Phys. Rev. 86, 52 (1952).
}

favorably oriented grains in the time of application of the load. The formation time of a slip band in the highly stressed grain would be considerably shorter than the loading time in the constant strain-rate tests of Suits and Low, according to the data of this investigation. Worthington and Smith observed slip bands which did not traverse the grains emanating from grain boundaries in their constant strain-rate tests. This can be the result of stress gradients in the very coarse grained specimens, rather than an indication of the relative importance of dislocation generation and dislocation mobility.

The present observations indicate that a stress significantly greater than the friction stress is required to initiate slip in a fully annealed grain. The number of slip lines formed from a fresh scratch is much greater than the number formed from the grain boundaries. A high percentage of scratched grains develops measurable slip bands, while only a very low percentage of unscratched grains shows any slip lines. The slip lines in unscratched grains are generally much longer than the ones formed in the scratched grains indicating that grain boundary sources are activated only in the most highly stressed grains.

(1) The velocity of screw dislocations in slip bands of silicon-iron follows the relation $v=v_{0}\left(\tau / \tau_{0}\right)^{n}$ with $v_{0}=$ $1 \mathrm{~cm} / \mathrm{sec}, \tau_{0}=2.30 \times 10^{9} \mathrm{dyn} / \mathrm{cm}^{2}$, and $n=30.1$. This is in good agreement with the measurements of Stein and Low ${ }^{1}$ on edge dislocations, and the observations of Low and Guard ${ }^{16}$ that screw dislocations have lower velocity than edge dislocations at the same stress. Elastic anisotropy has a significant effect on the dislocation velocity in polycrystalline grains of silicon-iron due to the high sensitivity of dislocation velocity to stress.

(2) Grain boundaries act as the source of slip bands in the relatively fine-grained $(76 \mu)$ polycrystalline silicon-iron used in this investigation.

(3) A stress significantly greater than the friction stress is required to initiate slip bands in a fully annealed grain.

\section{ACKNOWLEDGMENTS}

The advice and encouragement of Professor D. S. Wood and Professor D. S. Clark throughout the course of this work is gratefully acknowledged. 